\title{
Síndrome de Burnout em Professores: Prevalência e Fatores Associados
}

\author{
Mary Sandra Carlotto ${ }^{1}$ \\ Pontifíicia Universidade Católica do Rio Grande do Sul
}

\begin{abstract}
RESUMO - A Síndrome de Burnout é uma reação ao estresse excessivo relacionada ao trabalho. O objetivo do estudo foi identificar a prevalência da Síndrome de Burnout em 882 professores de escolas da região metropolitana de Porto Alegre-RS. Foram utilizados como instrumentos de pesquisa: um questionário elaborado especificamente para levantamento de variáveis demográficas, laborais e o MBI- Maslach Burnout Inventory (HSS-ED). Os resultados obtidos evidenciam 5,6\% de professores com alto nível de exaustão emocional, $0,7 \%$ em despersonalização e 28,9\% com baixa realização profissional. Mulheres, sem companheiro fixo, sem filhos, com idade mais elevada, que possuem maior carga horária, que atendem maior número de alunos e trabalham em escolas públicas apresentam maior risco de desenvolvimento de Burnout.
\end{abstract}

Palavras-chave: Síndrome de Burnout; prevalência; professores.

\section{The Burnout Syndrome in Teachers: Prevalence and Associated Factors}

\begin{abstract}
The burnout syndrome is a response to prolonged occupational stress. The aim of this study was to identify the prevalence of the Burnout Syndrome in 881 teachers from schools in the metropolitan region of Porto Alegre-RS. Two research instruments were used: a questionnaire to evaluate sociodemographic and professional variables and the MBI-Maslach Burnout Inventory (HSS-ED). Results showed that 5.6\% of the teachers showed high levels of emotional exhaustion, $0.7 \%$ depersonalization, and $28.9 \%$ low professional accomplishment. Older women without children, who are not engaged in a steady relationship, who have heavier workloads, teach lots of students and work in public schools show a higher risk of developing burnout.
\end{abstract}

Keywords: Burnout syndrome; prevalence; teachers

A categoria docente tem sido desde a fase pioneira de estudos sobre a Síndrome de Burnout (SB), uma das mais investigadas. Em 1979 há o primeiro registro de estudo descritivo realizado com professores (Perlman \& Hartman, 1982). Na década de 1980, cresce o interesse por Burnout, pois diversas investigações mostraram resultados considerados alarmantes. Foram identificados sintomas em grupos profissionais que, até então, não eram consideradas populações de risco, pelo contrário, por serem profissões consideradas vocacionais, sendo a docência uma delas, acreditava-se que esses profissionais obtinham gratificações em todos os níveis, dos pessoais aos sociais (Delgado et al., 1993).

No Brasil, a categoria docente, segundo análise da produção científica realizada por Carlotto e Câmara (2008), é uma das que contempla um maior número de investigações (Batista, Carlotto, Coutinho \& Augusto, 2010; Carlotto \& Palazzo, 2006; Carvalho, 1995; Codo, 1999; Levy, 2006; Mallar \& Capitão, 2004; Mazon, Carlotto \& Câmara, 2008; Moura, 1997; Silva \& Carlotto, 2003). No entanto, se comparada com a literatura internacional ainda é insuficiente em termos de consolidação de resultados.

A profissão docente é considerada pela Organização Internacional do Trabalho (OIT), como uma das mais estressantes, com forte incidência de elementos que conduzem à SB. Esse fenômeno, que atinge professores de diferentes países,

1 Endereço para correspondência: Av. Mauá, 645, apto 504, Centro São Leopoldo - RS. CEP 93110-320. Fone: (51) 3037.2530. E-mail: mscarlotto@pesquisador.cnpq.br parece portar um caráter epidêmico mundial que extrapola as fronteiras nacionais (Gil-Monte, 2008).

Atualmente, o ofício de professor, independente do nível de ensino em que atue tipo de escola, pública ou privada, está se configurando como uma profissão alvo de inúmeros estressores psicossociais presentes no seu contexto de trabalho. A atividade docente, entendida em tempos passados como uma profissão vocacional de grande satisfação pessoal e profissional tem dado lugar ao profissional de ensino excessivamente atrelado a questões tecnoburocráticas. Há uma redução da amplitude de atuação do trabalho, as tarefas de alto nível são transformadas em rotinas, há menos tempo para executar o trabalho, para atualização profissional, lazer e convívio social, bem como escassas oportunidades de trabalho criativo. Claramente, evidencia-se a existência de diversificação de responsabilidades com maior distanciamento entre a execução, realizada pelos professores, e o planejamento das políticas que norteiam seu trabalho, elaborado por outras pessoas. Neste modelo, os professores são mais técnicos do que profissionais (Kelchtermans, 1999). A esses aspectos somam-se os baixos salários e as precárias condições de trabalho (Leite \& Souza, 2007).

Na perspectiva pública, a categoria de professores sofre muitas críticas, é extremamente cobrada em seus fracassos e raramente é reconhecida por seu sucesso. Nenhuma categoria tem sido tão severamente avaliada e cobrada pela população em geral nas últimas décadas como a de professores (Farber, 1991). Entre as inúmeras demandas enfrentadas pelos professores, destaca-se a sobrecarga mental e a emocional. A 
função docente se caracteriza pela exigência de altos níveis de concentração, precisão e atenção diversificada. Do ponto de vista emocional, lhe é exigido envolvimento com os alunos, pais ou responsáveis, colegas e equipe técnica, relações estas que, em muitas ocasiões, podem ser ou tornar-se conflitivas (Salanova, Martínez \& Lorente, 2005), uma vez que estas não são escolhidas por ele e com freqüência não aceitam beneficiar-se ou reconhecer seus esforços (Pines \& Aronson, 1988).

Assim, a organização do trabalho do professor possui características que o expõem a fatores estressantes que, se persistentes, podem levá-lo a desenvolver a SB (Guglielmi \& Tatrow, 1998). Burnout é o resultado do estresse crônico, típico do cotidiano do trabalho, principalmente quando neste existem excessiva pressão, conflitos, poucas recompensas emocionais e pouco reconhecimento (Harrison, 1999), sendo considerado um fenômeno psicossocial constituído de três dimensões: Exaustão Emocional, Despersonalização e Baixa Realização Profissional. A Exaustão Emocional caracteriza-se por uma falta ou carência de energia e um sentimento de esgotamento emocional, sendo sua maior causa a sobrecarga de trabalho A Despersonalização ocorre quando o profissional passa a tratar os clientes, os colegas e a organização de forma distante e impessoal. Por fim, a Baixa Realização Profissional caracteriza-se por uma tendência do trabalhador em se auto-avaliar de forma negativa, sentindo-se insatisfeito com seu desenvolvimento profissional, experimentando um declínio no sentimento de competência e na sua capacidade de interagir com as pessoas (Maslach, Schaufeli \& Leiter, 2001).

O surgimento da SB é um processo paulatino e cumulativo, com incremento progressivo em termos de severidade (Delgado et al., 1993; Doménech, 1995). Sua evolução pode levar anos, até mesmo décadas (Rudow, 1999) e quase nunca é notada em seus estágios iniciais (Guimarães, 2000). Segundo Doménech (1995), no caso do professor, o processo é iniciado com uma sensação de inadequação na função e a percepção de ausência de recursos para enfrentar as exigências de seu trabalho. Há sensação de diminuição de sua capacidade de concentração, de resolver problemas e tomar decisões. Como conseqüência, o professor tende a aumentar o seu esforço, surgindo sinais evidentes de irritação, ansiedade, tensão, medo de não ter sucesso nas aulas, de manter a disciplina, com uma percepção exagerada de suas lacunas e dificuldades. Como forma de aliviar esses sentimentos e tentar adaptar-se pode desenvolver um distanciamento emocional associado a atitudes críticas e depreciativas sobre seu trabalho e alunos. Pode ocorrer somatizações que o levam a problemas médicos e não raras vezes ao afastamento no trabalho.

Embora a legislação brasileira já contemple a SB como doença relacionada ao trabalho, desde 1999, por meio do Decreto no 3048 de 06 de maio de 1999 do Ministério da Previdência e Assistência Social do Brasil (Ministério da Saúde, 2001), sua dimensão e caracterização específica ainda são pouco conhecidas (Carlotto \& Câmara, 2008).

Várias investigações sobre Burnout pontuam que a síndrome é resultante de fatores pessoais e contextuais, contudo, estudos apontam que aspectos contextuais, particularmente os relacionados ao trabalho, são mais associados a altos níveis de Burnout (Burke \& Greenglas, 1989; Grayson \& Alvarez,
2008; Klusmann, Kunter, Trautwein, Lüdtke \& Baumert, 2008; Lindblom, Linton, Fedeli \& Bryngelsson, 2006; Maslach \& Schaufeli, 1993; Maslach \& Godberg, 1998; Maslach, Schaufeli, \& Leiter, 2001; McCulloch \& O’Brien, 1986).

Estudos sobre a prevalência e fatores associados ao burnout, como variáveis sociodemográficas e laborais, realizados em diversos países ainda são contraditórios e necessitam ampliar suas investigações (Arce, Nakamura \& Jólluskin, 2003; Gil-Monte, 2005; Moriana-Elvira \& Herruzo-Cabrera, 2004). Essa questão ocorre devido a questões culturais de determinados países e categorias funcionais e também pelos diferentes pontos de corte utilizados, uma vez que nem todos os países que investigam burnout possuem validação dos mesmos.

Nesse sentido, este estudo epidemiológico observacional analítico de corte transversal (Grimes \& Shulz, 2002) procurou identificar a prevalência da SB e sua relação com fatores sociodemográficos e laborais em professores.

\section{Método}

\section{Participantes}

A amostra se constituiu de 881 professores que exercem atividade docente em oito escolas públicas e seis escolas privadas de médio porte localizadas na zona urbana de três cidades localizadas na região metropolitana de Porto Alegre - RS. Todas podem ser caracterizadas como de médio porte, possuem os três níveis de ensino e se equivalem quanto ao número de professores (35 a 68), e o de alunos (600 a 800). Foram excluídos os professores que exerciam somente atividades administrativas e os que estavam na instituição há menos de seis meses. Os professores investigados, em sua maioria, são mulheres $(54,1 \%)$, casados $(54,8 \%)$, possuem filhos $(75,6 \%)$ e apresentam idade média de 32,2 anos (DP=9,92). Possuem em média 11,5 anos de experiência profissional $(\mathrm{DP}=8,25)$, trabalham em média 30 horas $(\mathrm{DP}=12,5)$ e contatam diariamente uma média de 103,07 alunos (DP=98,59). Quanto ao tipo de escola, 60,8\% de professores atuam a maior parte de sua carga horária em escola pública e $39,2 \%$ em escola privada.

\section{Instrumentos}

Para o levantamento das variáveis demográficas (sexo, idade, situação conjugal, filhos) e laborais (carga horária, tempo de experiência de ensino, número de alunos que contata diariamente, tipo de escola) foi utilizado um questionário elaborado especificamente para este estudo baseado no referencial sobre a Síndrome de Burnout em professores. Para avaliar a SB foi utilizado o MBI-ED, Maslach Burnout Inventory - Educators Survey (Maslach \& Jackson, 1986), versão específica para professores, adaptada para o uso no Brasil por Carlotto e Câmara (2004). O inventário avalia como o trabalhador vivencia seu trabalho, de acordo com as três dimensões que constituem a síndrome: Exaustão Emocional, Despersonalização e Realização Profissional. 
Constitui-se de 22 itens que indicam a freqüência das respostas. Neste estudo, utiliza-se o sistema de pontuação adotado por Tamayo (1997) na adaptação brasileira do MBI-HSS, que considera 1 para "nunca"; 2 para "raramente"; 3 para "algumas vezes"; 4 para " com freqüência"; 5 para "sempre". Altos escores em Exaustão Emocional e Desepersonalização e baixos escores em Realização Profissional (esta subescala é inversa) são indicativos de Burnout (Maslach \& Jackson, 1981). Estudo realizado por Carlotto e Câmara (2004) indica que a versão brasileira do MBI apresenta os requisitos necessários em termos de consistência interna e validade fatorial para ser utilizada na avaliação da Síndrome de Burnout em professores em nossa realidade.

\section{Procedimentos}

Primeiramente foi realizado um contato com a direção de cada instituição de ensino, momento no qual foi apresentado o objetivo do estudo a fim de obter a autorização e o apoio para a aplicação dos instrumentos. Estes foram entregues pessoalmente aos professores, sendo a coleta realizada ao final da aplicação. Em algumas escolas a mesma foi realizada no intervalo de aula e em outras após reunião de professores. Foram realizados os procedimentos éticos conforme Resolução 196 do Conselho Nacional de Saúde (CNS). A pesquisa tem autorização do Comitê de Ética de afiliação da autora. Foi esclarecido aos professores e diretores das instituições de ensino tratar-se de uma pesquisa sem quaisquer efeitos avaliativos individuais e/ou institucionais e que as respostas seriam anônimas e confidenciais. O banco de dados foi digitado e posteriormente analisado através do SPSS 15.0 (Statistical Package for the Social Sciences). Primeiramente realizaram-se análises descritivas de caráter exploratório a fim de avaliar a distribuição dos itens, casos omissos ou possíveis erros de digitação.

Para cálculo da prevalência utilizou-se como critério de risco o ponto de corte sugerido por Shirom (1989) baseado na escala tipo Likert na qual os sujeitos atribuem os valores para os itens da escala. No caso da escala utilizada, definiu-se o ponto 4 que corresponde a resposta "com freqüência". Esta, segundo o autor, é uma alternativa válida para identificar os níveis de Burnout baseado na freqüência dos sintomas quando não existe no país de estudo pontos de corte baseados em percentis validados. Para analisar a magnitude e a direção do relacionamento entre as dimensões da SB e variáveis

Tabela 1. Valores dos níveis obtidos para as dimensões de Burnout com a amostra do estudo segundo critério de respostas da escala

\begin{tabular}{lccccccc}
\hline & \multicolumn{2}{c}{$\mathrm{M}<4$} & \multicolumn{2}{c}{$\mathrm{M} \geq \mathbf{4}$} & \multicolumn{2}{c}{ Total } \\
& $\mathrm{n}$ & $\%$ & $\mathrm{n}$ & $\%$ & $\mathrm{n}$ & $\%$ \\
\hline Dimensões & 832 & 94,4 & 49 & 5,6 & 881 & 100 \\
$\begin{array}{l}\text { Exaustão Emocional } \\
\text { Despersonalização }\end{array}$ & 875 & 99,3 & 6 & 0,7 & 881 & 100 \\
$\begin{array}{l}\text { Baixa Realização } \\
\text { Profissional }\end{array}$ & 626 & 71,1 & 255 & 28,9 & 881 & 100 \\
\hline
\end{tabular}

quantitativas utilizou-se a prova de correlação de Pearson e para avaliação da diferença entre variáveis qualitativas utilizou-se a prova $t$ de student.

\section{Resultados}

Em termos de prevalência, verifica-se que a dimensão com percentual mais elevado é a de Baixa Realização Profissional e a de menor percentual é a de Despersonalização.

Resultados obtidos identificaram que quanto maior a idade dos professores, maior é o sentimento de distanciamento e menor o de realização no trabalho. Com relação à carga horária, verifica-se que quanto mais elevada maior é o sentimento de desgaste emocional e menor o sentimento de realização com o trabalho. A elevação do número de alunos atendidos diariamente aumenta o desgaste emocional, o distanciamento e diminui a realização profissional, conforme demonstrado na Tabela 2.

Análise realizada revela que as mulheres apresentam maior exaustão emocional e maior realização no trabalho, sendo que homens apresentam maior despersonalização. Quanto à situação conjugal, verifica-se que sujeitos sem companheiro fixo possuem maior realização no trabalho. Professores sem filhos possuem maior exaustão emocional e os que têm filhos menor despersonalização e realização no trabalho. Os que atuam em escolas públicas possuem maior desgaste emocional, maior despersonalização e menor realização profissional.

\section{Discussão}

A SB ocorre pelo desenvolvimento conjunto de baixa realização profissional e exaustão emocional, tendo como estratégia de enfrentamento a despersonalização (Gil-Monte, Peiró \& Valcárcel, 1998; Gil-Monte, 2005). Considerando o processo de desenvolvimento do Burnout proposto, os resultados obtidos no presente estudo apontam para a possibilidade deste encontrar-se em curso. Verifica-se $28,9 \%$ de professores com alto nível de baixa realização profissional, 5,6\% com alta exaustão emocional e $0,7 \%$ com despersonalização. Quanto à prevalência, os percentuais são bastante inferiores

Tabela 2. Associação entre dimensões de Burnout e variáveis quantitativas

\begin{tabular}{lccc}
\hline Variáveis & $\mathrm{EE}$ & $\mathrm{DE}$ & $\mathrm{RP}$ \\
\hline Idade & $-0,013$ & $-0,069^{*}$ & $-0,006$ \\
Carga Horária & $0,073^{*}$ & 0,049 & $-0,506^{* *}$ \\
Alunos dia & $0,116^{* *}$ & $0,110^{* *}$ & $-0,266^{* *}$ \\
Tempo de docência & $-0,043$ & $-0,032$ & $-0,056$ \\
\hline
\end{tabular}

Nota: $* \mathrm{p}<0,05$

** $\mathrm{p}<0,01$

EE: Exaustão emocional DE: Despersonalização RP: Realização Profissional 
Tabela 3. Associação entre dimensões de Burnout e variáveis qualitativas

\begin{tabular}{|c|c|c|c|c|c|c|c|c|c|c|}
\hline \multirow[t]{2}{*}{ Dimensões } & \multicolumn{4}{|c|}{$\mathrm{EE}$} & \multicolumn{3}{|c|}{$\mathrm{DE}$} & \multicolumn{3}{|c|}{$\mathrm{RP}$} \\
\hline & $\mathrm{N}$ & $\mathrm{M}$ & DP & $p$ & $\mathrm{M}$ & DP & $p$ & $\mathrm{M}$ & DP & $p$ \\
\hline \multicolumn{11}{|l|}{ Sexo } \\
\hline Feminino & 474 & 2,40 & 0,89 & 0,00 & 1,47 & 0,54 & 0,03 & 3,52 & 1,32 & 0,00 \\
\hline Masculino & 403 & 2,20 & 0,78 & & 1,56 & 0,64 & & 3,03 & 1,36 & \\
\hline \multicolumn{11}{|c|}{ Situação conjugal } \\
\hline Sem comp. & 394 & 2,30 & 0,91 & 0,91 & 1,59 & 0,57 & 0,71 & 3,48 & 1,24 & 0,00 \\
\hline Com comp. & 477 & 2,31 & 0,84 & & 1,49 & 0,61 & & 3,14 & 1,43 & \\
\hline \multicolumn{11}{|l|}{ Filhos } \\
\hline Não & 215 & 2,50 & 0,86 & 0,00 & 1,44 & 1,14 & 0,03 & 2,73 & 0,92 & 0,00 \\
\hline Sim & 666 & 2,24 & 0,87 & & 1,28 & 1,15 & & 3,46 & 0,51 & \\
\hline \multicolumn{11}{|c|}{ Tipo de escola } \\
\hline Pública & 535 & 2,40 & 0,94 & 0,00 & 1,56 & 0,66 & 0,08 & 2,03 & 0,74 & 0,00 \\
\hline Privada & 346 & 2,16 & 0,66 & & 1,45 & 0,49 & & 4,10 & 1,13 & \\
\hline
\end{tabular}

Nota: EE: Exaustão emocional DE: Despersonalização RP: Realização Profissional

ao encontrados em estudo nacional realizado com professores por Codo (1999). Naquele estudo o autor registrou que $48,4 \%$ da categoria demonstram estar com pelo menos uma das três dimensões do burnout alterada, estando $25,1 \%$ com alta exaustão emocional, 10,7\% com despersonalização e $31,9 \%$ denotam falta de envolvimento com as tarefas do magistério. Investigação recente desenvolvida por Batista, Carlotto, Coutinho e Augusto (2010), com professores de João Pessoa, identificou resultados também superiores, $33,6 \%$ dos professores apresentaram alto nível de Exaustão Emocional, 8,3\% alto nível de Despersonalização e 43,4\% baixo nível de Realização Profissional. Estudo desenvolvido com professores portugueses, realizado por Gomes, Silva, Mourisco, Silva, Mota e Montenegro (2006) revela 14\% com alto nível de exaustão, 17,9\% com despersonalização e 6\% não realizados profissionalmente. Rudow (1999), ao avaliar Burnout em professores germânicos e americanos, encontrou diferentes resultados nas três dimensões, Germânicos eram menos afetados pela exaustão emocional (23\%) comparados aos americanos (33\%). Somente 8\% dos germânicos apresentavam níveis altos na dimensão despersonalização, sendo que os americanos apresentavam 33\%. Sentimento de realização no trabalho era de $40 \%$ para os germânicos e em 35\% para os americanos. Assim, verifica-se a existência de expressiva variação entre os resultados obtidos, provavelmente relacionados a questões culturais e também de critérios para definição de pontos de corte, uma vez que muitos estudos utilizam pontos de corte estadunidense, o que se mostra incorreto para o uso em outras populações (Gil-Monte, 2005).

A possibilidade de existência de um processo em curso, também pode estar relacionada a duas outras questões: a primeira delas diz respeito à dimensão de despersonalização que pode não estar representando a realidade, ou seja, baixos valores. Segundo Farber (1991), muitas pessoas podem não ter condições de reconhecer, verdadeiramente, o que está acontecendo com elas próprias, o que dificultaria a identificação de questões relacionadas a essa dimensão. Assim, pode-se pensar na ocorrência de alguma dificuldade para o professor revelar certas atitudes suas no trabalho, tais como não tratar seus alunos com afetividade, uma vez que essa é uma importante expectativa de pais, administração escolar e sociedade em geral, fazendo parte do perfil idealizado do professor (Carlotto \& Palazzo, 2006). A segunda questão é a situação peculiar do estudo transversal em epidemiologia ocupacional que exclui o possível doente, levando ao "efeito do trabalhador sadio" (Mc Michael, 1976). Todos os respondentes foram avaliados em seu local de trabalho, em pleno exercício profissional. Essa é uma situação que pode subestimar o tamanho dos riscos oferecidos pelo processo de trabalho, porque os mais afetados não conseguem manter-se no emprego, afastando-se por licenças para tratamento de saúde, por demissões e por outros motivos. A partir dessas ponderações, o resultado obtido enseja preocupação, tendo em vista que os professores estão em pleno exercício funcional, provavelmente, agravando seu quadro com sérios prejuízos para a qualidade de seu trabalho com conseqüências importantes na relação ensino-aprendizagem.

Burnout em professores é um fenômeno complexo, multidimensional, resultante da interação entre aspectos individuais e o ambiente de trabalho (Kelchtermans, 1999). Os resultados obtidos no estudo corroboram essa afirmação na medida em que variáveis demográficas e laborais apresentaram associação com as dimensões da Síndrome

Quanto às variáveis demográficas, o resultado com relação ao sexo segue a tendência de outros estudos nacionais (Carlotto, 2003; Mallar \& Capitão, 2004) e internacionais (Burke, Greenglass \& Schwarzer 1996; Gomes et al., 2006; Vercambre, Brosselin, Gilbert, Nerrière \& Kovess-Masféty, 2009) que apontam as mulheres com maior exaustão emocional, menor despersonalização e maior sentimento de realização profissional. Segundo Maslach e Jackson (1985), a diferença de gênero pode estar relacionada a três questões: responsabilidade familiar, tipo de ocupação e papel do sexo na socialização. A última questão abordada é a mais importante, pois identificam as idéias de um envolvimento maior das mulheres com cuidados, alimentação e preocupação com 
o bem-estar de outras pessoas. A profissão docente ainda é percebida como uma extensão da função materna. A elevação de exaustão emocional por parte das mulheres pode ser interpretada também pela questão da emocionalidade vinculada ao papel feminino. Maslach (1999) também referem à dupla jornada exercida pela mulher uma das razões para a diferença com relação à exaustão emocional. Já o sentimento de não realização profissional nos homens pode estar relacionado às expectativas de sucesso, competição e desenvolvimento, geralmente elementos mais identificados com o papel masculino (Maslach \& Jackson, 1985).

Com relação à idade, verifica-se que quanto mais jovem, maior é o sentimento de distanciamento das pessoas com as quais o professor tem que se relacionar em seu trabalho. $\mathrm{O}$ mesmo resultado foi encontrado em estudo realizado com professores por Correia, Gomes e Moreira (2010). Jovens profissionais geralmente precisam aprender a lidar com as demandas do trabalho (Gavish \& Friedman, 2010; Maslach,1982), desenvolver habilidades e maturidade relacional (Cherniss,1980). Este resultado também pode relacionar-se à preparação insuficiente para a prática de trabalho docente Rudow (1999) que na sua formação contempla de forma intensiva questões pedagógicas, deixando em segundo plano questões relacionadas ao contato interpessoal e gestão grupal. Farber (1991) acredita que jovens professores possuem maior risco de desenvolver Burnout, provavelmente pelas expectativas irrealistas em relação à profissão. Geralmente professores iniciam suas carreiras bastante entusiasmados e com muita dedicação, tendo senso do significado social do seu trabalho e imaginando que o mesmo lhe proporcionará grande satisfação pessoal. Contudo, as inevitáveis dificuldades do ensino em interação com vulnerabilidades e questões pessoais, acrescidas das pressões e valores sociais, engendram os sentimentos de frustração, desencadeando uma série de questionamentos com relação à profissão e ao desejo de manter o investimento na mesma.

Resultados obtidos quanto à situação conjugal revelam que participantes sem companheiro fixo possuem maior realização no trabalho. Professores sem filhos possuem maior exaustão emocional, maior despersonalização e menor realização no trabalho. Maslach e Jackson (1985) e Ozdemir (2007) verificaram que as pessoas casadas apresentavam menos Burnout que as solteiras, separadas ou viúvas. Maslach e Jackson (1985) interpretam esse dado entendendo que as pessoas casadas geralmente são mais maduras psicologicamente e possuem um estilo de vida mais estável. Outra questão levantada pelas autoras é que estas pessoas possuem uma visão diferente de seu trabalho. Para elas, o salário, os benefícios e a segurança são fatores mais importantes que entusiasmo e satisfação pessoal. Uma terceira questão abordada é sobre a relação entre variáveis familiares e a SB, ou seja, a vida familiar pode ter propiciado mais experiências em lidar com outras pessoas e seus problemas. As pessoas podem não ter muita experiência de contato com público em seu trabalho, mas podem tê-la desenvolvido em função de sua vida familiar. Neste sentido teriam mais paciência e mais equilíbrio em lidar com situações de crise.

$\mathrm{Na}$ análise das dimensões de Burnout e associação com variáveis laborais, verifica-se que quanto mais elevada a carga horária, maior o sentimento de desgaste emocional e menor o sentimento de realização com o trabalho. Resultado semelhante foi identificado em estudo com professores por Carlotto e Palazzo (2006). A elevação do número de alunos atendidos diariamente aumenta o desgaste emocional (Manassero et al., 2005; Rudow,1999), o distanciamento e diminui a realização profissional (Russell, Altmaier \& Van Velzen, 1987). Muitas horas de trabalho são correlacionadas com atitudes negativas principalmente quando envolve contato direto e contínuo com as pessoas (Maslach, 1976). Quando o número de clientes aumenta, também se elevam as demandas, e, se estas são mais contínuas do que intermitentes, o trabalhador pode ficar mais vulnerável ao Burnout (Cordes \& Dougherty, 1993). No caso do professor, problemas na relação com os alunos são um dos principais preditores da SB (Otero et al., 2008; Pas, Bradshaw, Hershfeldt \& Leaf, 2010; Rudow, 1999; Vercambre et al., 2009; Yong \& Yue, 2008). Relacionada a essa questão, é apontada a falta de reciprocidade com os alunos (Bakker et al., 2000; Schaufeli, 2005), a ambigüidade, o conflito e a sobrecarga de papel (Farber, 1991; Konukman et al., 2010). O professor com maior carga horária, geralmente também tem um maior número de classes e alunos para atender, o que pode conduzir a uma sobrecarga de trabalho e sentimento de que o mesmo não é fonte de realização. Segundo Manassero et al. (2005), a falta de equilíbrio entre as exigências da profissão docente e as recompensas recebidas como acompanhar o progresso dos alunos e ter o reconhecimento dos mesmos relacionam-se ao desenvolvimento da SB.

Professores que atuam em escola pública apresentam maiores médias nas três dimensões da síndrome Burnout relacionadas a diversos estressores, alguns típicos da natureza da função exercida, outros provenientes do contexto institucional e social onde ela é exercida. Diferenças entre os contextos de trabalho, considerando-se uma mesma profissão são responsáveis, em grande medida, pela forma como se configura a síndrome de Burnout (Guglielmi \& Tatrow, 1998). Professores de escolas públicas e privadas possuem atribuições semelhantes, decorrentes da natureza da sua função. No entanto, existem aspectos contextuais e institucionais da realidade educacional brasileira, que conduz a vivências e práticas diferenciadas. Determinados fatores de estresse laboral presentes na profissão docente, provavelmente ganham intensidade diferenciada em função dos contextos onde emergem. Fatores de estresse como condições salariais, condições físicas e pedagógicas, apoio técnico, perfil de clientela, expectativa de pais e comunidade, entre outros, são percebidos de forma diferenciada. Segundo Romeu (1987), os problemas no campo da educação no Brasil são mais sérios em relação à escola pública do que em relação à particular e, mesmo entre estas, mostram-se mais acentuados em umas do que em outras. As escolas particulares, embora integrantes do sistema de ensino em suas diretrizes e bases mais gerais, mantêm certa autonomia interna que lhes garante algumas vantagens em termos de trabalho. Por questões mercadológicas há uma tendência a atrair clientes-alunos através de estratégias de marketing, investimento em equipamentos sofisticados, da modernização de laboratórios, da ampliação e do conforto de suas instalações (Moura, 1997). De acordo com Andrade, Nunes, Neto e Abramovay (2004), no Brasil, de um modo geral, os professores da escola pública estão em condições econômicas e sociais inferiores aos das escolas privadas. 
Identificar o perfil de risco e os sinais precoces de desenvolvimento do Burnout é de fundamental importância para intervenções preventivas (Maslach \& Leiter, 2008). O estudo aponta para um perfil de risco constituído de professores jovens, sem relacionamento conjugal estável e filhos, que atuam em escolas públicas, possuem elevada carga horária de trabalho e relacionam-se com um maior número de alunos.

Deve-se ter cautela com relação aos resultados obtidos, uma vez que esses são decorrentes de instituições localizadas em uma região específica do país, não sendo, portanto, passíveis de generalizações para outras instituições ou profissionais. A investigação sugere a necessidade de aprofundamento dos resultados obtidos, uma vez que a literatura brasileira ainda é incipiente em termos de resultados de Burnout nessa categoria profissional. Assim, sugere-se a realização de novos estudos, com inclusão de outras variáveis e delineamentos em contextos diferenciados.

\section{Referências}

Andrade, E. R., Nunes, M. F. R., Neto, M., F., \& Abramovay, M. (2004). O Perfil dos professores brasileiros: o que fazem o que pensam, o que almejam / Pesquisa Nacional UNESCO. São Paulo: Moderna.

Arce, R., Nakamura, A. P., \& Jólluskin, G. (2003). Burnout: estudio del campo y nível de desempenho. Encuentros en Psicologia Social, 1(4), 35-39.

Bakker, A. B., Schaufeli, W. B., Demerouti, E., Janssen, P. P. M., Van Der Hulst, R., \& Brouwer, J. (2000). Using equity theory to examine the difference between burnout and depression. Anxiety, Stress, and Coping, 13, 247-268.

Batista, J. B. V., Carlotto, M. S., Coutinho, A. S., \& Augusto, L. G. da S. (2010). Prevalência da Síndrome de Burnout e fatores sociodemográficos e laborais em professores de escolas municipais da cidade de João Pessoa, PB. Revista Brasileira de Epidemiologia, 13(3), 502-512.

Burke, R. J., \& Greenglass E. R. (1989). Psychological burnout among men and women in teaching: an examination of the Cherniss model. Human Relations, 42(3), 261-273.

Burke, R. J., Greenglass, E. R., \& Schwarzer, R. (1996). Predicting teacher burnout over time: effects of work stress, social support and self-doubts on burnout and its consequences. Anxiety, Stress and Coping, 9(3), 261-275.

Carlotto, M. S. (2003). Síndrome de Burnout e gênero em docentes de instituições particulares de ensino. Revista de Psicologia da Universidade do Contestado, 1(1). Retrieved from http:// www.nead.uncnet.br/revista/psicologia

Carlotto, M. S., \& Câmara, S. G. (2004). Análise fatorial do Malasch Burnout Inventory (MBI) em uma amostra de professores de instituições particulares. Psicologia em Estudo, 9(3), 499-505.

Carlotto, M. S., \& Câmara, S. G. (2008). Análise da produção científica sobre a Síndrome de Burnout no Brasil. PsicoPUCRS, 39(2), 152-158.

Carlotto, M. S., \& Palazzo, L. dos S. (2006). Síndrome de burnout e fatores associados: um estudo epidemiológico com professores. Cadernos de Saúde Pública, 22(5),1017-1026.

Carvalho, M. M. B. (1995). O professor: Um profissional, sua saúde e a educação em saúde na escola. Tese de Doutorado, Universidade de São Paulo, São Paulo.
Cherniss, C. (1980). Professional burnout in human service organizations. New York: Praeger.

Codo, W. (1999). Educação: Carinho e trabalho. Petrópolis: Vozes.

Cordes, C. L., \& Dougherty, T. W. (1993). A review and integration of research on job burnout. Academy of Management Review, 18(4), 632- 636.

Correia, T., Gomes, A. R., \& Moreira, S. (2010). Stresse ocupacional em professores do Ensino Básico: um estudo sobre as diferenças pessoais e profissionais. In Actas do VII Simpósio Nacional de Investigação em Psicologia (pp.1477-1493). Associação Portuguesa de Psicologia, Lisboa, PO.

Delgado, A. C., Fuentes, J. M. B., Quevedo, M. P. A., Salgado, A. R., Sánchez, A. C., Sanchez, T. S., Velasco, C. A., \& Yela, J. R. B. (1993). Revisión teórica del burnout o desgaste profesional en trabajadores de la docencia. Caesura, 2, 47-65.

Doménech, B. D. (1995). Introduccion al sindrome "burnout" en profesores y maestros y su abordaje terapeutico. Psicologia Educativa, 1(1), 1-16.

Farber, B. A. (1991). Crisis in education. Stress and burnout in the American teacher. San Francisco: Jossey-Bass Inc.

Gavish, B., \& Friedman, I. A. (2010). Novice teachers' experience of teaching: a dynamic aspect of burnout. Social Psychology of Education, 13(2), 141-167.

Gil-Monte, P. R. (2005). El syndrome de quemarse por el trabajo (burnout). Una enfermidad laboral en la sociedad del bienestar. Madrid: Pirámide.

Gil-Monte, P. R. (2008). El síndrome de quemarse por el trabajo (burnout) como fenómeno transcultural. Informació Psicológica, 91-92, 4-11.

Gil-Monte, P. R., Peiró, J. M., \& Valcárcel, P. (1998). A model of burnout process development: An alternative from appraisal models of stress. Comportamento Organizacional e Gestão, 4(1), 165-179.

Gomes, R. A., Silva, M. J., Mourisco, S., Silva, S., Mota, A., \& Montenegro, N. (2006). Problemas e desafios no exercício da actividade docente: um estudo sobre o estresse, burnout, saúde física e satisfação profissional em professores do $3^{\circ}$ ciclo e ensino secundário. Revista Portuguesa de Educação, 19(1), 67-93.

Grayson, J. L., \& Alvarez, H. K. (2008). School climate factors relating to teacher burnout: a mediator model. Teaching and Teacher Education, 24, 1349-1363

Grimes, D. A., \& Shulz, K. F. (2002). An overview of clinical research: the lay of the land. The Lancet, 359, 57-61.

Guimarães, L. A. M. (2000). Saúde mental, estresse e qualidade de vida no trabalho. In J. C. Souza, L. A. M. Guimarães \& S. Grubits (Eds.), Interdisciplinaridade em saúde mental (pp.1719). Campo Grande: UCDB.

Guglielmi, R. S., \& Tatrow, K. (1998). Occupational stress, burnout, and health in teachers: a methodological and theoretical analysis. Review of Educational Research, 68(1), 61-69.

Harrison, B. J. (1999). Are you to burn out? Fund Raising Management, 30(3), 25-28.

Kelchtermans, G. (1999). Teaching career: between burnout and fading away? Reflections from a narrative and biographical perspective. In R. Vanderbergue \& M. A. Huberman (Eds.), Understanding and preventing teacher burnout: a source book of international practice and research (pp. 176-191). Cambridge: Cambridge University Press. 
Klusmann, U., Kunter, M., Trautwein, U., Lüdtke, O., \& Baumert, J. (2008). Engagement and emotional exhaustion in teachers: does the school context make a difference? Applied Psychology: An International Review, 57(Suppl.), 127-151.

Konukman, F., Agbuğa, B., Erdoğan, S., Zorba, E., Demirhan, G., \& Y1lmaz, I. (2010). Teacher-coach role conflict in school-based physical education in USA: a literature review and suggestions for the future. Biomedical Human Kinetics, 2, 19- 24.

Leite, M de P., \& Souza, A. N. de (2007). Condições do trabalho e suas repercussões na saúde dos professores da educação básica no Brasil - Estado da Arte. São Paulo: Fundacentro/Unicamp.

Levy, G. C. T. de M. (2006). Avaliar a sindrome de Burnout em professores da rede pública de ensino. Dissertação de Mestrado, Universidade do Estado do Rio de Janeiro, Rio de Janeiro.

Lindblom, K. M., Linton, S. J., Fedeli, C., \& Bryngelsson, I-L. (2006). Burnout in the Working Population: Relations to Psychosocial Work Factors. International Journal of Behavioral Medicine, 13(1), 51 - 59.

Mallar, S. C., \& Capitão, C. G. (2004). Burnout e hardiness: Um estudo de evidência de validade. Psico-USF, 9(1), 19-29.

Manassero, M. A., García, E., Torrens, G., Ramis, C., Vázquez, A., \& Ferrer, V. A. (2005). Burnout en la enseñanza: aspectos atribucionales. Revista de Psicología del Trabajo y de las Organizaciones, 21(1-2), 89-105.

Maslach, C. (1976). Burned-out. Human Behavior, 5(9), 26-22.

Maslach, C. (1982). Burnout: The cost of caring. Englewood Cliffs, N.J.: Prentice - Hall.

Maslach, C. (1999). Progress in understanding teacher burnout. In R. Vandenberghe \& A. M. Huberman (Eds.), Understanding and preventing teacher burnout (pp. 211-222). New York: Cambridge University Press.

Maslach, C., \& Goldberg, J. (1998). Prevention of burnout: news perspectives. Applied \& Preventive Psychology, 7, 63-74.

Maslach, C., \& Jackson, S. E. (1981). The measurement of experienced burnout. Journal of Occupational Behavior, 2, 99-113.

Maslach, C., \& Jackson, S. E. (1985). The role of sex and family variables in burnout. Sex Roles, 12(7/8), 837- 851.

Maslach, C., \& Jackson, S. E. (1986). Maslach Burnout Inventory. $2^{\text {nd }}$ ed. Palo Alto, CA: Consulting Psychologist Press.

Maslach, C., \& Leiter, M. P. (2008). Early predictors of job burnout and engagement. Journal of Applied Psychology, 93(3), 498-512.

Maslach, C., \& Schaufeli, W. B. (1993). Historical and conceptual development of burnout. Em W.B. Schaufeli, C. Maslach \& T. Marek (Eds.), Professional burnout: recent developments in theory and research (pp.1-16). New York: Taylor \& Francis.

Maslach, C., Schaufeli, W. B., \& Leiter, M. P. (2001). Job burnout. Annual Review Psychology, 52, 397-422.

Mazon, V., Carlotto, M. S., \& Câmara, S. G. (2008). Síndrome de Burnout e estratégias de coping em professores de escolas municipais da área urbana de uma cidade do litoral norte do Rio Grande do Sul. Arquivos Brasileiros de Psicologia, 60(1), 55-66.

McCulloch, A., \& O'Brien, L. (1986). The organizational determinants of worker burnout. Children \& Youth Services Review, 8(2), 175-190.
Mc Michael, A. J. (1976). Standardized mortality ratios and the healthy worker effect: scratching beneath the surface. Journal Occupational Medical, 18, 165-168.

Ministério da Saúde (2001). Doenças relacionadas ao trabalho: manual de procedimentos para os serviços de saúde. Brasília: Ministério da Saúde; OPAS/OMS.

Moriana-Elvira, J. A., \& Herruzo-Cabrera, J. (2004). Estrés y burnout en profesores, International Journal of Clinical and Health Psychology, 4(3), 597-621.

Moura, E. P. G. (1997). Saúde mental e trabalho. Esgotamento profissional em professores da Rede de Ensino Particular de Pelotas - RS. Dissertação de Mestrado, Pontifícia Universidade Católica do Rio Grande do Sul, Porto Alegre, RS.

Otero, L. J. M., Santiago, M. J., Godás, A., Castro, C., Villaedefrancos, E., \& Ponte, D. (2008). An integrative approach to burnout in secondary school teachers: examining the role of student disruptive behavior and disciplinary issues. International Journal of Psychology and Psychological Therapy, 8(2), 259-270.

Ozdemir, Y. (2007). The role of classroom management efficacy in predicting teacher burnout. International Journal of Social Sciences, 2(4), 257-263.

Pas, E. T., Bradshaw, C. P., Hershfeldt, P. A., \& Leaf, P. J. (2010). A multilevel exploration of the influence of teacher efficacy and burnout on response to student problem behavior and schoolbased service use. School Psychology Quarterly, 25(1), 13-27.

Perlman, B., \& Hartman A. E. (1982). Burnout: Sumary and future research. Human Relations, 35(4), 283-305.

Pines, A., \& Aronson, E. (1988). Career burnout. Causes and cures. New York: The Free Press.

Romeu, S. A. (1987). Escola: objetivos organizacionais e objetivos educacionais. São Paulo: EPU.

Rudow, B. (1999). Stress and burnout in the teaching profession: European studies, issues, and research perspectives. In R. Vanderbergue \& M. A. Huberman (Eds.), Understanding and preventing teacher burnout: a source book of international practice and research (pp.38-58). Cambridge: Cambridge University Press.

Russell, D. W., Altmaier, E., \& Van Velzen, D. (1987). Job-related stress, social support, and burnout among classroom teachers. Journal of Applied Psychology, 72(2), 269-273.

Salanova, M., Martínez, I. M., \& Lorente, L. (2005). ¿Cómo se relacionan los obstáculos y facilitadores organizacionales con el burnout docente?: Un estúdio longitudinal. Revista de Psicología del Trabajo y de las Organizaciones, 21(1-2), 37-54.

Schaufeli, W. B. (2005). Burnout en profesores: una perspectiva social del intercambio. Revista de Psicología del Trabajo y de las Organizaciones, 21(1-2), 15-35.

Shirom, A. (1989). Burnout in work organizations. In C. L. Cooper \& I. Robertson (Eds.), International review of industrial an organizational psychology (pp. 25-48). New York: Wiley \& Sons.

Silva, G. N., \& Carlotto M. S. (2003). Síndrome de Burnout: um estudo com professores da rede pública. Revista de Psicologia Escolar e Educacional, 7(2), 145-153.

Tamayo, R. N. (1997). Relação entre a Síndrome de Burnout e os valores organizacionais no pessoal de enfermagem de dois Hospitais Públicos. Dissertação de Mestrado, Universidade de Brasília, Brasília. 
Vercambre, M. N., Brosselin, P., Gilbert, F., Nerrière, E., \& KovessMasféty, V. (2009). Individual and contextual covariates of burnout: a cross-sectional nationwide study of French teachers. BMC Public Health, 9, 333. Retrieved from http://www. biomedcentral. com/1471-2458/9/333

Yong, Z., \& Yue, Y. (2008). Causes for burnout among secondary and elementary school teachers and preventive strategies.

Recebido em 12.05.2009

Primeira decisão editorial em 22.09.2010

Versão final em 25.06.2011

Chinese Education and Society, 40(5), 78-85.

Aceito em 15.09.2011

\section{Ciclo de Estudos Sobre o Imaginário \\ "Imaginário e Dinâmicas do Segredo"}

21 de Outubro de 2011

Recife, PE, BR 